\title{
Molecular determinants of heart failure with normal left ventricular ejection fraction
}

\author{
Attila Borbély ${ }^{1,2}$, Zoltán Papp ${ }^{1}$, István Édes ${ }^{1}$, Walter J. Paulus ${ }^{2}$ \\ ${ }^{1}$ Division of Clinical Physiology, Institute of Cardiology, University of Debrecen, Medical and Health Science \\ Center, Móricz Zsigmond 22, H-4032 Debrecen, Hungary \\ ${ }^{2}$ Department of Physiology, Institute for Cardiovascular Research, VU University Medical Center, \\ Van der Boechorststraat 7, 1081BT Amsterdam, The Netherlands
}

Correspondence: Attila Borbély, e-mail: borbelya@dote.hu

\begin{abstract}
:
In population-based studies, heart failure with normal left ventricular (LV) ejection fraction (HFNEF) is now increasingly recognized and referred to as diastolic heart failure. However, the pathogenic mechanisms underlying HFNEF are incompletely understood, mainly because of limited availability of human myocardial biopsy material. Nevertheless, recent studies have examined in vivo hemodynamics, in vitro cardiomyocyte function, myofilamentary protein composition, collagen content and deposition of advanced glycation end products from LV endomyocardial biopsies. These measures were compared between HFNEF patients, subjects without symptoms of heart failure (controls), patients with heart failure and reduced ejection function (HFREF), and patients with HFNEF and HFREF with diabetes mellitus. This article summarizes the various findings of these studies and focuses on the possible correlations among altered LV myocardial structure, cardiomyocyte function, myofilamentary proteins, and extracellular matrices. These findings revealed novel mechanisms responsible for diastolic LV dysfunction, and they have important therapeutic implications, particularly HFNEF, for which a specific heart failure treatment strategy is largely lacking.
\end{abstract}

Key words: heart failure, diastolic function, cardiomyocytes, myofilamentary proteins, fibrosis, diabetes mellitus

\begin{abstract}
Abbreviations: AGEs - advanced glycation end products, CVF - collagen volume fraction, ECM - extracellular matrix, HFNEF - heart failure with normal ejection fraction, HFREF heart failure with reduced ejection fraction, LVEF - left ventricular ejection fraction, PKA - protein kinase A
\end{abstract}

\section{Introduction}

Heart failure with normal ejection fraction (HFNEF) is currently diagnosed in approximately half of heart failure patients [11]. The majority of HFNEF patients are elderly women with a history of systolic hypertension, many with left ventricular (LV) hypertrophy, obesity, and diabetes [10, 23, 30,33]. Despite the increased recognition of $\mathrm{LV}$ diastolic dysfunction and HFNEF, the pathophysiology of this disease remains controversial. Furthermore, evidence-based guidelines for management of HFNEF are largely lacking [21]. Many basic questions remain unanswered on the mechanisms underlying LV diastolic dysfunction. Impaired LV relaxation [22], decreased LV distensibility, increased LV end-diastolic stiffness [3, 45, 46], and pericardial and right ventricular constraint $[32,34]$ have all been implicated as contributing factors to $\mathrm{LV}$ 
diastolic dysfunction. However, the most probable scenario is that diastolic dysfunction integrates abnormalities of the extracellular matrix (ECM), cardiomyocytes and their myofilamentary proteins [20] (Fig. 1B-D). Nevertheless, the relative contribution of these factors to diastolic indices of LV function in vivo (Fig. 1A) is obscure. Failure to resolve these controversies mainly arises from the difficulties in obtaining human biopsy and/or necropsy materials [20, 37], which would allow clinical and hemodynamic features to be correlated with cellular and molecular myocardial properties. The relative involvement of the aforementioned elements was recently examined in in vitro studies using LV endomyocardial biopsies of HFNEF patients. This review summarizes the differences in LV myocardial structure and function and recapitulates the suggested novel mechanisms responsible for the abnormal diastolic LV function.

\section{Clinical characteristics and in vivo hemodynamics}

As proposed in the consensus statement by the Heart Failure and Echocardiography Associations of the European Society of Cardiology, the diagnosis of HFNEF requires the following conditions to be satisfied: 1) signs or symptoms of heart failure; 2) normal or mildly abnormal systolic LV function; 3) evidence of diastolic LV dysfunction [35]. Normal or mildly abnormal systolic LV function implies both a left ventricular ejection fraction (LVEF) of greater than $50 \%$ and an LV end-diastolic volume index (LVEDVI) of less than $97 \mathrm{~mL} / \mathrm{m}^{2}$. Diagnostic evidence of diastolic LV dysfunction can be obtained invasively (LV enddiastolic pressure (LVEDP) $>16 \mathrm{mmHg}$ or mean pulmonary capillary wedge pressure $>12 \mathrm{mmHg}$ ) or

\section{A LV pressure-volume relation}

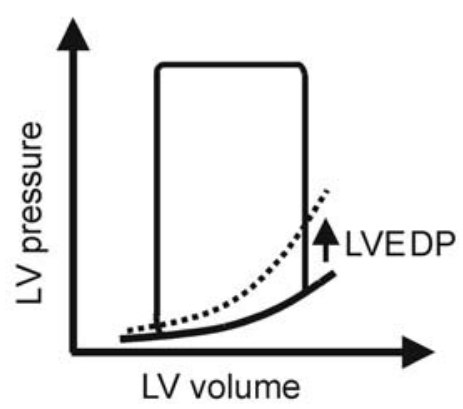

B Extracellular matrix

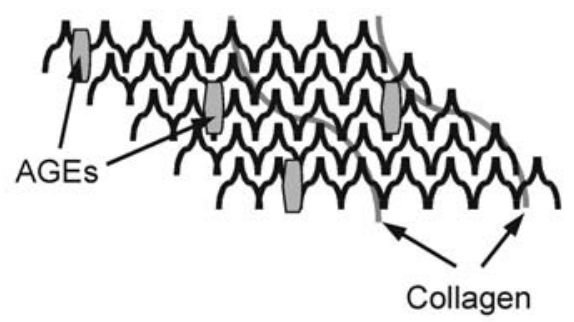

C Cardiomyocyte

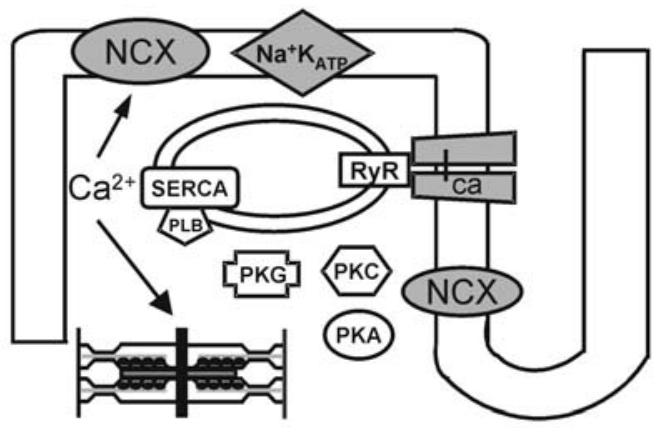

D Myofilaments

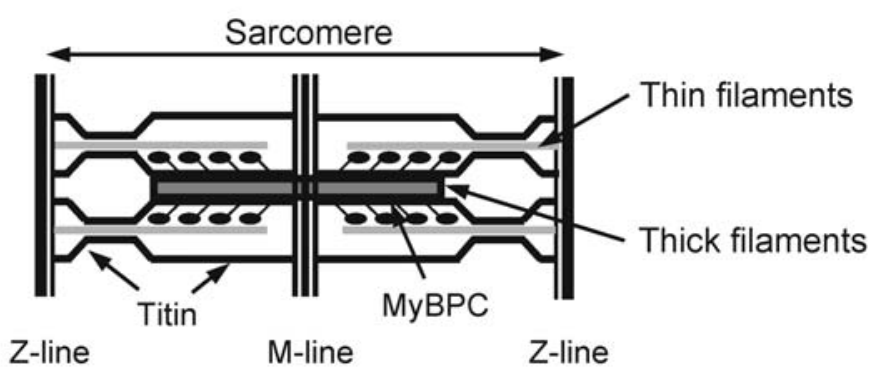

Fig. 1. Determinants of HFNEF. (A) LV diastolic pressure-volume relation in HFNEF is shifted upward when measured in vivo, indicating an increase in LVEDP. (B) The altered diastolic properties are coupled with changes in the ECM that surrounds each myocyte. Collagen can also be post-translationally cross-linked and alter properties of the ECM by protein-glucose interaction (generation of AGEs). (C) At the level of the cardiomyocyte, calcium handling and signaling proteins are particularly important. Changes in the expression of the $\mathrm{Na}^{+} / \mathrm{Ca}^{2+}$ exchanger ( $\mathrm{NCX}$ ), $\mathrm{Ca}^{2+}$ uptake proteins (PLB, SERCA), sarcoplasmic reticular $\mathrm{Ca}^{2+}$ channel (RyR) and ion pumps $\left(\right.$ e.g., $\mathrm{Na}^{+} / \mathrm{K}^{+}$ATPase, $\left.\mathrm{NK}_{\text {ATP }}\right)$ and their posttranslation modifications by several protein kinases (PKA, PKC, PKG) can alter their activity. (D) At the level of the myofilaments, alterations in proteins within the thick and thin filaments, myosin-binding protein C (MyBPC), and the giant cytoskeletal protein titin can lead to abnormal cardiomyocyte stiffness and relaxation 
non-invasively by tissue Doppler (TD) (E/E'> 15). The transvascular catheter technique used in our recent studies $[6,39,40]$ allowed us to assess hemodynamic characteristics in each individual patient at the time of cardiac catheterization and biopsy sample retrieval. Invasive measurements combined with echocardiography revealed a significantly higher LV peak systolic pressure (LVPSP), LVEDP, circumferential LV end-diastolic wall stress $(\sigma)$ and radial myocardial stiffness modulus (SM) in the HFNEF patient group compared to similar measures in the control group [6]. The increased LVEDP and $\sigma$ with comparable LVEDVI indicated a reduced LV diastolic distensibility, and the increased SM suggested increased myocardial stiffness in the HFNEF patient group. By comparing clinical characteristics of HFNEF patients with HFREF patients, we found that more HFNEF patients had a history of arterial hypertension and were obese [39]. LVPSP, LVEF, cardiac index, deceleration time and SM were all higher in HFNEF patients. In addition, we found that diabetic HFNEF and HFREF patients had reduced LV diastolic distensibility and a higher SM compared to non-diabetic patients [40]. Moreover, LVMI was higher in HFREF and HFNEF patients compared to the control population. The elevated LVMI in HFREF patients was believed to be a compensatory mechanism to preserve LV wall thickness with the larger LVEDVI. However, in HFNEF patients, the elevated LVMI resulted in significant increases in LV wall thickness and LVMI/LVEDVI ratio. These findings indicated that eccentric LV hypertrophy occurs in HFREF, while HFNEF could be characterized by concentric LV hypertrophy.

\section{Level of ECM: fibrosis and advanced glycation end (AGE) product deposition}

Diastolic properties are frequently linked to characteristics of the ECM that surrounds each myocyte and forms bundles among muscle fibers. Connective tissue consists of perimysial fibers enveloping groups of myocytes, smaller endomysial fibers supporting and connecting individual cells, and endomysial weaves enveloping individual myocytes [20]. Many studies have suggested the importance of myocardial fibrosis in diastolic dysfunction [25, 27]. Moreover, recent studies have begun to target signaling factors for fi- brosis [13, 24, 31] and correlate changes in collagen/fibrosis with chamber stiffening. A direct link between fibrosis and stiffness may seem to be well established, yet controversy exists. Not all quantitative assessments of fibrosis in larger animal models of heart failure and in humans have identified correlations between stiffness and collagen content. In our recent studies, histological analysis of human endomyocardial biopsies revealed higher collagen volume fractions (CVF) in HFNEF patients than in controls [6]. HFNEF patients were equally distributed over low, intermediate and high CVF classes. In low and intermediate CVF classes, the elevated indices of diastolic function (LVEDP, $\sigma$ and SM), despite low levels of interstitial fibrosis, indicated that CVF is not the sole contributor to diastolic LV dysfunction.

Increased diastolic LV stiffness is recognized as the earliest manifestation of LV dysfunction induced by diabetes mellitus, and it frequently becomes the main functional deficit of the diabetic heart [12, 15, 19]. However, as shown in our comparative histological study, the mechanisms underlying the increased diastolic LV stiffness differ between HFREF and HFNEF patients. Deposition of AGEs and collagen were found to be important determinants of the increased LV stiffness in diabetic patients with HFREF, whereas high cardiomyocyte resting tension $\left(\mathrm{F}_{\text {passive }}\right)$ was likely responsible for the increased LV stiffness in diabetic patients with HFNEF [40]. Deposition of AGEs with concomitant protein/glucose interaction results from long-standing hyperglycemia and affects diastolic LV stiffness through direct and indirect mechanisms $[1,20,36]$. AGE cross-linking of collagen increases its tensile strength, and this altered biophysical property of collagen increases diastolic LV stiffness. In addition, AGE deposition can indirectly augment diastolic LV stiffness through enhanced collagen formation and reduced nitric oxide bioavailability. Indeed, enhanced collagen formation in the presence of AGEs was confirmed in our recent study [40], and low myocardial nitric oxide bioavailability was previously demonstrated in HFREF patients [4, 5]. Postmyocarditis patients with HFREF frequently show signs of persistent myocardial microvascular inflammation [38]. This inflammation may facilitate AGE deposition [4] and may explain the preferential AGE deposition in small intramyocardial vessels of diabetic patients with HFREF. The clinical importance of endothelial AGE deposition was recently confirmed in hypertensive patients who showed im- 
proved endothelial function with a cross-link breaker [43]. We observed higher CVF values in diabetic than in non-diabetic HFREF patients [40]. Activation of fibroblasts in diabetic HFREF patients may also result from the aforementioned AGE deposition, protein kinase $\mathrm{C}$ activation, cyclooxygenase- 2 up-regulation, or high intracellular glucose concentrations $[1,3,8]$.

\section{Cardiomyocyte level: structural and functional alterations}

\section{Increased cardiomyocyte diameter and Z-line thickness in HFNEF}

Basic studies have provided histological evidence of cardiomyocyte hypertrophy in HFNEF [39, 40]. The increased myocyte diameter (MyD) in HFNEF and the marginally increased MyD in HFREF resemble the previously reported cardiomyocyte remodeling in concentric and eccentric LV hypertrophy [16]. In concentric LV hypertrophy, cardiomyocytes grow in a transverse direction while maintaining a constant cell length, whereas in eccentric hypertrophy, cardiomyocytes grow proportionally in longitudinal and transverse directions. This difference in cardiomyocyte remodeling correlates with distinct patterns of peptide growth factor induction in both conditions [9]. As shown in our studies, different correlation exists between cardiomyocyte hypertrophy and fibrosis in HFREF and HFREF patients [39]. The increased
MyD in HFREF was accompanied by collagen deposition. In HFNEF, collagen deposition was associated with a similar further increase in MyD. For each level of CVF, MyD in HFNEF patients exceeded MyD in HFREF patients. This excess cardiomyocyte hypertrophy in HFNEF was likely related to a history of arterial hypertension, which was present in $73 \%$ of the HFNEF patients and only $13 \%$ of the HFREF patients.

Cardiac hypertrophy has also been reported in experimental models of diabetes mellitus and insulin resistance [18]. However, we previously noted Z-line widening in humans as well as an associated elevation in $F_{\text {passive }}$ [40]. Therefore, we suggested that Z-line widening is associated with alterations in the elastic properties of cytoskeletal proteins, which pull at and open up adjacent $\mathrm{Z}$ lines.

\section{Increased cardiomyocyte stiffness in HFNEF}

The relative importance of $\mathrm{F}_{\text {passive }}$ for $\mathrm{LV}$ myocardial stiffness was also determined [6, 39]. LV endomyocardial catheter biopsies ( $5 \mathrm{mg}$ wet weight) procured from control subjects and patients with HFNEF were used for isolation of single cardiomyocytes. The resting tension $\left(\mathrm{F}_{\text {passive }}-\right.$ tension determined in $\mathrm{Ca}^{2+}$-free solution) of the cardiomyocytes was measured to detect diastolic dysfunction at the level of cardiomyocytes. When cardiomyocytes derived from HFNEF patients were stretched to a sarcomere length of $2.2 \mu \mathrm{m}, \mathrm{F}_{\text {passive }}$ was found to be twice as high as that of control cardiomyocytes [6] (Fig. 2). Treatment with protein kinase A (PKA) induced a significant drop in
A

B

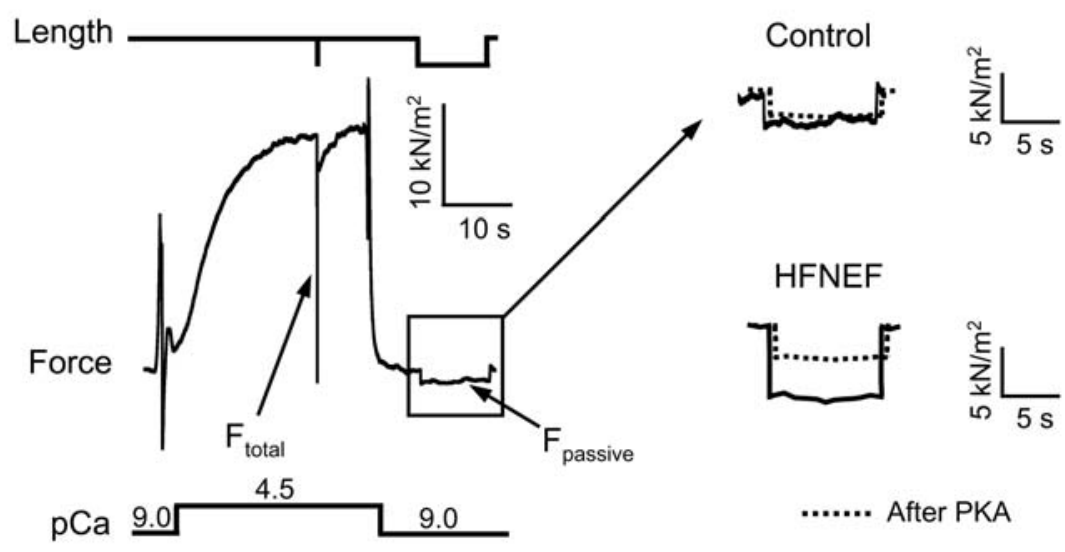

Fig. 2. Cardiomyocyte stiffening in HFNEF. (A) Representative force recording during contraction-relaxation cycle obtained in a single cardiomyocyte during maximal activation ( $\mathrm{pCa} 4.5$ ). (B) $\mathrm{F}_{\text {passive }}$ determined in $\mathrm{Ca}^{2+}$-free solution (pCa 9) was significantly higher in HFNEF than in control cardiomyocytes. In vitro administration of PKA (dotted line) reduced the elevated $F_{\text {passive }}$ of HFNEF cardiomyocytes to the level observed in the control group (the difference between $F_{\text {total }}$ and $F_{\text {passive }}$ is the $\mathrm{Ca}^{2+}$-activated force, see text and ref. 6 for more details) 
$\mathrm{F}_{\text {passive }}$ in cardiomyocytes from HFNEF patients, whereas $\mathrm{F}_{\text {passive }}$ in control cardiomyocytes was unaltered [6]. In a follow-up study, $\mathrm{F}_{\text {passive }}$ of HFREF cardiomyocytes was found to be significantly lower than that of HFNEF cardiomyocytes; however, PKA had a similar effect on $F_{\text {passive }}$ of HFREF cardiomyocytes [39].

\section{Changes in the myofilamentary protein compo- sition and phosphorylation}

During isolation of cardiomyocytes, endomysial collagen structures are removed; therefore, a high $\mathrm{F}_{\text {passive }}$ in cardiomyocytes of HFNEF patients can only result from increased stiffness of the cardiomyocytes themselves. Because cardiomyocytes were also incubated in solution supplemented with Triton X-100, the integrity of sarcolemmal and sarcoplasmic membranes was disrupted. Under these conditions, disturbed calcium handling (because of modified expression and/or phosphorylation of sarcoplasmic reticular $\mathrm{Ca}^{2+}$-ATPase [44], phospholamban [17, 28], sarcoplasmic calcium release channels [29] and sodium/calcium exchangers [41]) was also ruled out as a possible cause of the observed elevation in $\mathrm{F}_{\text {passive }}$. Therefore, the increased cardiomyocyte stiffness must be attributed to alterations of myofilament or cytoskeletal proteins. However, no differences were found between the HFNEF and control groups in the expression of cardiac sarcomeric proteins, such as myosin heavy chain, actin, TnT, TnI, desmin and tropomyosin [6]. Moreover, degradation of several contractile proteins, including TnI, was ruled out in both the control and HFNEF groups by western immunoblot analysis. Therefore, it was unlikely that a change in isoform composition or protein degradation accounted for the high $F_{\text {passive }}$ of cardiomyocytes observed in the HFNEF group. However, the high $F_{\text {passive }}$ could result from hypophosphorylation of the sarcomeric target proteins of PKA, which include TnI, myosin binding protein-C, and/or titin. Consistent with this proposal, PKA-mediated phosphorylation of the elastic N2B spring element of titin was shown to reduce diastolic stiffness in isolated rat cardiomyocytes [42]. However, the ratio of dephosphorylated to total TnI did not differ between the cardiac tissues of patients from the control and the HFNEF groups [6], so the fall in $\mathrm{F}_{\text {passive }}$ induced by in vitro PKA exposure likely resulted from the correction of a phosphorylation deficit of myosin binding protein- $\mathrm{C}$ or titin.
Future studies are clearly required to identify the hypophosphorylated myofilamentary protein(s) responsible for the high cardiomyocyte $F_{\text {passive }}$ in HFNEF. These hypophosphorylated sarcomeric proteins, together with ECM modifications, could then be considered myocardial targets for drug therapy of HFNEF. Whether other protein kinases (PKC, PKD or $\mathrm{PKG}$ ) are also able to reduce cardiomyocyte $\mathrm{F}_{\text {passive }}$ in HFNEF cardiomyocytes or not also remains to be determined.

\section{Correlations between in vivo and in vitro data}

The transvascular catheter techniques have allowed for assessment of in vivo hemodynamic characteristics and for correlation with functional parameters measured in vitro [6]. When $\mathrm{F}_{\text {passive }}$ values of control and HFNEF cardiomyocytes were pooled, significant correlations were found between $\mathrm{F}_{\text {passive }}$ and indices of diastolic LV dysfunction, such as LVEDP, SM and $\sigma$. The relations between $F_{\text {passive }}$ and indices of diastolic LV function all leveled off at higher values of LVEDP, $\sigma$, and $\mathrm{E}$. This could result from diuretic therapy to compensate patients before catheterization or from more intense interstitial fibrosis at severe diastolic LV dysfunction. Endomyocardial biopsy samples from patients with HFNEF had higher CVF than did controls, and in a bivariate linear regression analysis, both $\mathrm{F}_{\text {passive }}$ and CVF were significantly correlated with LVEDP and $\sigma$. A predominant effect of interstitial fibrosis upstream of diastolic LV dysfunction is consistent with previous experimental studies, which showed diastolic muscle stiffness to originate from structures within the sarcomere for sarcomere lengths up to $2.2 \mu \mathrm{m}$ [26] and from perimysial fibers once filling pressures exceeded $30 \mathrm{mmHg}$ [14].

\section{Conclusions}

Because HFNEF patients can have subtle abnormalities of systolic LV function and HFREF patients can have diastolic LV dysfunction, the clinical classification of heart failure patients into HFNEF and HFREF 
phenotypes is challenging. Recent basic studies compared human LV myocardium of HFNEF and HFREF patients and revealed significant differences at the structural and functional levels. Moreover, in the absence of coronary artery disease, the mechanisms responsible for the elevated diastolic LV stiffness also differed between diabetic HFREF and HFNEF patients. The distinct structural and functional abnormalities support HFNEF and HFREF as separate heart failure phenotypes. These mechanistic basic studies suggest that the reduction of high cardiomyocyte resting tension could serve as a potential therapeutic target for HFNEF patients.

\section{Acknowledgments}

Attila Borbély is a recipient of a Mecenatúra Grant from the UDMHSC, Hungary (Mec-4/2008) and of a basic research fellowship grant from the Heart Failure Association of the European Society of Cardiology, Nice, France. Zoltán Papp holds Bolyai Fellowship of the Hungarian Academy of Sciences. Support by grant ETT 449/2006 is gratefully appreciated. There are no conflicts of interest to be reported.

\section{References:}

1. Asbun J, Villarreal FJ: The pathogenesis of mocardial fbrosis in the setting of diabetic cardiomyopathy. J Am Coll Cardiol, 2006, 47, 693-700.

2. Bagi Z, Erdei N, Papp Z, Edes I, Koller A: Up-regulation of vascular cyclooxygenase-2 in diabetes mellitus. Pharmacol Rep, 2006, 58, Suppl, 52-56.

3. Baicu CF, Zile MR, Aurigemma GP, Gaasch WH: Left ventricular systolic performance, function, and contractility in patients with diastolic heart failure. Circulation, 2005, 111, 2306-2312.

4. Baidoshvili A, Krijnen PAJ, Kupreishvili K, Ciurana C, Bleeker W, Nijmeijer R, Visser CA et al.: $\mathrm{N}^{\varepsilon}$-(carboxymethyl)lysine depositions in intramyocardial blood vessels in human and rat acute myocardial infarction: a predictor or reflection of infarction? Arterioscler Thromb Vasc Biol, 2006, 26, 2497-2503.

5. Bauersachs J, Widder JD: Endothelial dysfunction in heart failure. Pharmacol Rep, 2008, 60, 119-126.

6. Borbely A, van der Velden J, Papp Z, Bronzwaer JGF, Edes I, Stienen GJM, Paulus WJ: Cardiomyocyte stiffness in diastolic heart failure. Circulation, 2005, 111, 774-781.

7. Bronzwaer JGF, Heymes C, Visser CA, Paulus WJ: Myocardial fibrosis blunts nitric oxide synthase-related preload reserve in human dilated cardiomyopathy. Am J Physiol Heart Circ Physiol, 2003, 284, H10-H16.

8. Brownlee M: The pathobiology of diabetic complications: a unifying mechanism. Diabetes, 2005, 54, $1615-1625$
9. Calderone A, Takahashi N, Izzo NJ, Jr., Thaik CM, Colucci WS: Pressure- and volume-induced left ventricular hypertrophies are associated with distinct myocyte phenotypes and differential induction of peptide growth factor mRNAs. Circulation, 1995, 92, 2385-2390.

10. Chen HH, Lainchbury JG, Senni M, Bailey KR, Redfield MM: Diastolic heart failure in the community: clinical profile, natural history, therapy, and impact of proposed diagnostic criteria. J Card Fail, 2002, 8, 279-287.

11. Cleland JGF, Cohen-Solal A, Aguilar JC, Dietz R, Eastaugh J, Follath F, Freemantle N et al.: Management of heart failure in primary care (the IMPROVEMENT of Heart Failure Programme): an international survey. Lancet, 2002, 360, 1631-1639.

12. Diamant M, Lamb HJ, Groeneveld Y, Endert EL, Smit JWA, Bax JJ, Romijn JA et al.: Diastolic dysfunction is associated with altered myocardial metabolism in asymptomatic normotensive patients with wellcontrolled type 2 diabetes mellitus. J Am Coll Cardiol, 2003, 42, 328-335.

13. Diez J, Querejeta R, Lopez B, Gonzalez A, Larman M, Martinez Ubago JL: Losartan-dependent regression of myocardial fibrosis is associated with reduction of left ventricular chamber stiffness in hypertensive patients. Circulation, 2002, 105, 2512-2517.

14. Factor SM, Flomenbaum M, Zhao MJ, Eng C, Robinson TF: The effects of acutely increased ventricular cavity pressure on intrinsic myocardial connective tissue. J Am Coll Cardiol, 1988, 12, 1582-1589.

15. Fang ZY, Prins JB, Marwick TH: Diabetic cardiomyopathy: evidence, mechanisms, and therapeutic implications. Endocr Rev, 2004, 25, 543-567.

16. Gerdes AM: Cardiac myocyte remodeling in hypertrophy and progression to failure. J Card Fail, 2002, 8, S264-S268.

17. Hasenfuss G, Pieske B: Calcium cycling in congestive heart failure. J Mol Cell Cardiol, 2002, 34, 951-969.

18. Ilercil A, Devereux RB, Roman MJ, Paranicas M, O'Grady MJ, Lee ET, Welty TK et al.: Associations of insulin levels with left ventricular structure and function in American Indians: the strong heart study. Diabetes, 2002, 51, 1543-1547.

19. Jain A, Avendano G, Dharamsey S, Dasmahapatra A, Agarwal R, Reddi A, Regan T: Left ventricular diastolic function in hypertension and role of plasma glucose and insulin. Comparison with diabetic heart. Circulation, 1996, 93, 1396-1402.

20. Kass DA, Bronzwaer JGF, Paulus WJ: What mechanisms underlie diastolic dysfunction in heart failure? Circ Res, 2004, 94, 1533-1542.

21. Katz AM, Zile MR: New molecular mechanism in diastolic heart failure. Circulation, 2006, 113, 1922-1925.

22. Kitzman DW: Diastolic heart failure in the elderly. Heart Fail Rev, 2002, 7, 17-27.

23. Klapholz M, Maurer M, Lowe AM, Messineo F, Meisner JS, Mitchell J, Kalman J et al.: Hospitalization for heart failure in the presence of a normal left ventricular ejection fraction: Results of the New York heart failure registry. J Am Coll Cardiol, 2004, 43, 1432-1438. 
24. Kuwahara F, Kai H, Tokuda K, Kai M, Takeshita A, Egashira K, Imaziumi T: Transforming growth factor- $\beta$ function blocking prevents myocardial fibrosis and diastolic dysfunction in pressure-overloaded rats. Circulation, 2002, 106, 130-135.

25. Lamberts RR, Willemsen MJJM, Perez NG, Sipkema P, Westerhof N: Acute and specific collagen type I degradation increases diastolic and developed tension in perfused rat papillary muscle. Am J Physiol Heart Circ Physiol, 2004, 286, H889-H894.

26. Linke WA, Popov VI, Pollack GH: Passive and active tension in single cardiac myofibrils. Biophys J, 1994, 67, 782-792.

27. MacKenna DA, Omens JH, McCulloch AD, Covell JW: Contribution of collagen matrix to passive left ventricular mechanics in isolated rat hearts. Am J Physiol Heart Circ Physiol, 1994, 266, H1007-H1018.

28. MacLennan DH, Kranias EG: Phospholamban: a crucial regulator of cardiac contractility. Nat Rev Mol Cell Biol, 2003, 4, 566-577.

29. Marks AR, Reiken S, Marx SO: Progression of heart failure: Is protein kinase A hyperphosphorylation of the ryanodine receptor a contributing factor? Circulation, 2002, 105, 272-275.

30. Masoudi FA, Havranek EP, Smith G, Fish RH, Steiner JF, Ordin DL, Krumholz HM: Gender, age, and heart failure with preserved left ventricular systolic function. J Am Coll Cardiol, 2003, 41, 217-223.

31. Matsumoto T, Wada A, Tsutamoto T, Ohnishi M, Isono T, Kinoshita M: Chymase inhibition prevents cardiac fibrosis and improves diastolic dysfunction in the progression of heart failure. Circulation, 2003, 107, 2555-2558.

32. Morris-Thurgood JA, Frenneaux MP: Diastolic ventricular interaction and ventricular diastolic filling. Heart Fail Rev, 2000, 5, 307-323.

33. Owan TE, Hodge DO, Herges RM, Jacobsen SJ, Roger VL, Redfield MM: Trends in prevalence and outcome of heart failure with preserved ejection fraction. N Engl J Med, 2006, 355, 251-259.

34. Pak PH, Maughan WL, Baughman KL, Kass DA: Marked discordance between dynamic and passive diastolic pressure-volume relations in idiopathic hypertrophic cardiomyopathy. Circulation, 1996, 94, 52-60.

35. Paulus WJ, Tschope C, Sanderson JE, Rusconi C, Flachskampf FA, Rademakers FE, Marino P et al. How to diagnose diastolic heart failure: a consensus statement on the diagnosis of heart failure with normal left ventricular ejection fraction by the Heart Failure and Echocardiography Associations of the European Society of Cardiology. Eur Heart J, 2007, 28, 2539-2550.
36. Poornima IG, Parikh P, Shannon RP: Diabetic cardiomyopathy: the search for a unifying hypothesis. Circ Res, 2006, 98, 596-605.

37. Redfield MM: Understanding "diastolic" heart failure. N Engl J Med, 2004, 350, 1930-1931.

38. Vallbracht KB, Schwimmbeck PL, Kuhl U, Rauch U, Seeberg B, Schultheiss HP: Differential aspects of endothelial function of the coronary microcirculation considering myocardial virus persistence, endothelial activation, and myocardial leukocyte infiltrates. Circulation, 2005, 111, 1784-1791.

39. van Heerebeek L, Borbely A, Niessen HWM, Bronzwaer JGF, van der Velden J, Stienen GJM, Linke WA et al.: Myocardial structure and function differ in systolic and diastolic heart failure. Circulation, 2006, 113, 1966-1973.

40. van Heerebeek L, Hamdani N, Handoko ML, FalcaoPires I, Musters RJ, Kupreishvili K, Ijsselmuiden AJ et al.: Diastolic stiffness of the failing diabetic heart: importance of fibrosis, advanced glycation end products, and myocyte resting tension. Circulation, 2008, 117, $43-51$.

41. Weber CR, Piacentino V, III, Houser SR, Bers DM: Dynamic regulation of sodium/calcium exchange function in human heart failure. Circulation, 2003, 108, 2224-2229.

42. Yamasaki R, Wu Y, McNabb M, Greaser M, Labeit S, Granzier H: Protein kinase A phosphorylates titin's cardiac-specific N2B domain and reduces passive tension in rat cardiac myocytes. Circ Res, 2002, 90, 1181-1188.

43. Zieman SJ, Melenovsky V, Clattenburg L, Corretti MC, Capriotti A, Gerstenblith G, Kass DA: Advanced glycation end product crosslink breaker (alagebrium) improves endothelial function in patients with isolated systolic hypertension. J Hypertens, 2007, 25, 577-583.

44. Zile MR, Baicu CF, Bonnema DD: Diastolic heart failure: definitions and terminology. Prog Cardiovasc Dis, 2005, 47, 307-313.

45. Zile MR, Baicu CF, Gaasch WH: Diastolic heart failure - abnormalities in active relaxation and passive stiffness of the left ventricle. N Engl J Med, 2004, 350, 1953-1559.

46. Zile MR, Brutsaert DL: New concepts in diastolic dysfunction and diastolic heart failure: Part I: diagnosis, prognosis, and measurements of diastolic function. Circulation, 2002, 105, 1387-1393.

Received:

September 22, 2008; in revised form: January 15, 2009. 